\title{
The Fast and Furious, a Rare Case of Primary Burkitt's Lymphoma of the Skull Base
}

Case

Rosla, Luqman', Goh, Bee-See ${ }^{2}$,Zahedi, Farah Dayana ${ }^{3}$, Kew, Thean Yean ${ }^{4}$, Abu

Report

Bakar, Azizi ${ }^{5}$

${ }^{1,2,3}$ Otolaryngology, National University Of Malaysia

${ }^{4}$ Radiology, National University Of Malaysia

${ }^{5}$ Neurosurgery, National University Of Malaysia

\begin{abstract}
Burkitt's Lymphoma of the head and neck region commonly involves the facial bones in its endemic variant and Waldeyer's ring in the sporadic variant. Base of skull is a rare site for primary Burkitt's lymphoma, with nasal and neuro-ophthalmology symptoms as its presentation. We report a case of a three year-old girl presented with sudden and progressive bilateral eye blindness associated with bilateral inward squint, proptosis and nasal blockage. Imaging study demonstrated a large anterior skull base lesion with extension into ethmoid and sphenoid sinuses. Tumor was removed via open surgery and histopathological examination confirmed the diagnosis of Burrkitt's lymphoma. Patient had permanent bilateral blindness postoperatively and succumbed two months after the initial presentation due to the agressiveness of the tumor. The late presentation, aggressiveness of the disease and lack of financial support contribute to her untimely demise. Burkitt's Lymphoma of the base of skull warrants early tissue diagnosis by means of surgical intervention to ensure prompt commencement of treatment.
\end{abstract}

Key Words: Blindness, Burkitt's Lymphoma, nasal blockage, skull base,

Received: 4 March 2018, Accepted: 27-May-2018

Corresponding Author: Goh, Bee-See, Tel.: +60193209305, E-mail: irenegbs@yahoo.com

ISSN: 2090-0740, June 2018, Vol.19, No.2

\section{INTRODUCTION}

Burkitt's lymphoma(BL) is an aggressive B-cell nonHodgkin lymphoma ${ }^{[1]}$. It is the fastest growing tumor where the cells doubles in 24-48 hours ${ }^{[2]}$. The etiological factor associated with BL is Ebstein Barr virus (EBV). The infected B-cell undergone chromosomal translocation that activates an oncogenes (c-MYC), and initiate the tumerogenesis ${ }^{[1,2]}$.

WHO has classified BL into three variants which are endemic, sporadic and immunodeficiency-related. Endemic type is related to malaria endemicity and it is commonly associated with EBV. The sporadic variant rarely associated with EBV and it occur throughout the world mainly in North Africa and Europe. In immunodeficiency-related variant, it is frequently seen in HIV infected patient with EBV association ${ }^{[1]}$.

In the endemic type of BL, head and neck involvement commonly affect the facial bones, where else involvement of Waldeyer's ring (palatine and lingual tonsils, nasopharyngeal adenoid) and nodal involvement are commonly seen in sporadic form ${ }^{[4]}$. Base of skull is a rare site for primary extranodal head and neck involvement of BL. We report a case of primary Burkitt's lymphoma of base of skull with nasal and neuroophthalmologic presentation.

\section{CASE REPORT}

A 3-year-old girl from Indonesia, presented with sudden progressive bilateral vision loss in three days associated bilateral inward squint, proptosis, nasal blockage and discharge. She had no fever, vomiting, epistaxis, neck swelling, weight loss or night sweats. Patient first presented to a local hospital in that country but no active management was done. Subsequently she was referred to our centre due to persistent pain around the nose and forehead after three weeks.

Clinical examination revealed bilateral proptosis, non reactive dilated pupils with no light perception and complete ophthalmoplegia (Figure 1). Other cranial nerves were intact and there was no cervical lymphadenopathy. There was difficulty to assess the function of olfactory nerve in this girl.

Magnetic Resonance imaging (MRI) demonstrated an 


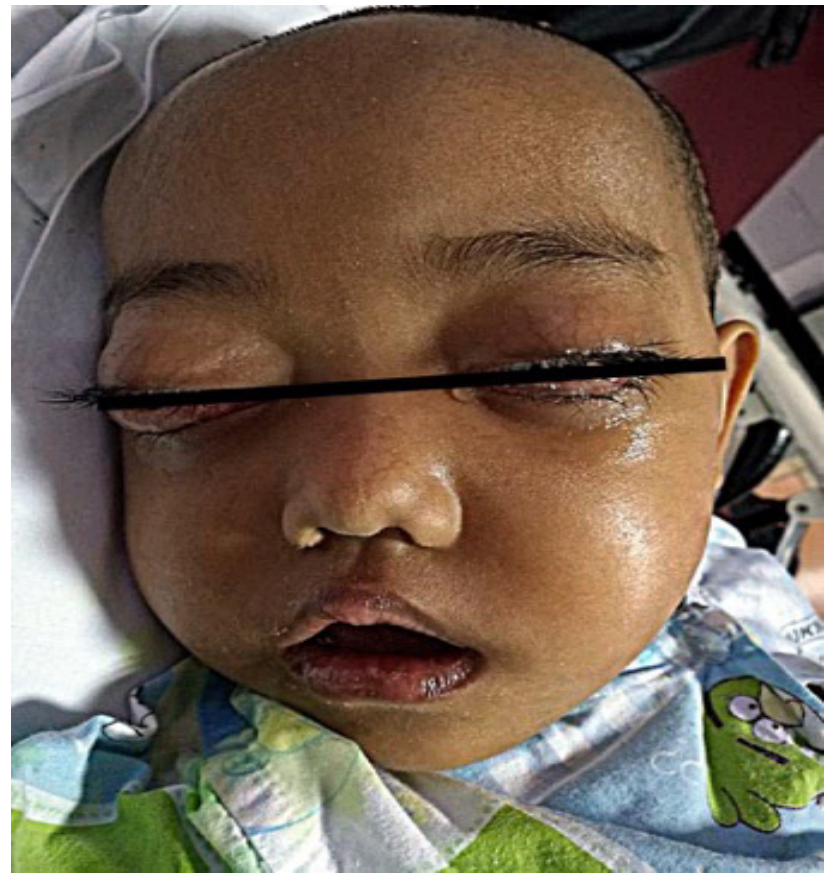

Fig. 1: Clinical examination of the patient showing bilateral proptosis.

anterior skull base tumor with epicenter at ethmoid sinus, involving the basi sphenoid. The tumor extends laterally intraorbitally through eroded lamina papyracea. Bilateral optic nerves were effaced by the tumor posteriorly and there was involvement of olfactory fossa (Figure 2 and 3 ).

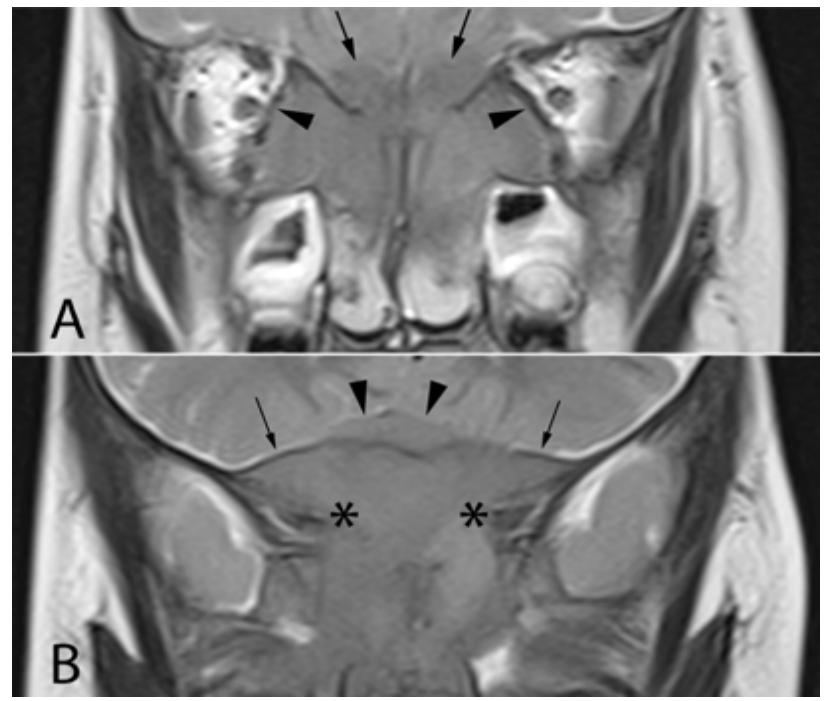

Fig. 2: (A) T2 weighted coronal MR image, slice positioned at the level of the cribriform plate. The epicentre of the mass is at the ethmoid air cells. It extends laterally intraorbitally through eroded laminae papyracea. Orbital fat is replaced medially, with no separation discernible between the mass and the medial recti muscles (arrowheads). At the anterior skull base, the mass has involved the olfactory fossae (arrows. Note that the olfactory bulbs normally visualised here are replaced by tumour signal intensity). No parenchymal oedema of the frontal lobes is observed.

(B) Part of superior extent of the mass centrally (arrowheads) has breached the dural lining (arrows), reaching the intradural compartment. The intracanalicular segments of bilateral optic nerves (asterisks) are encroached upon by tumour.

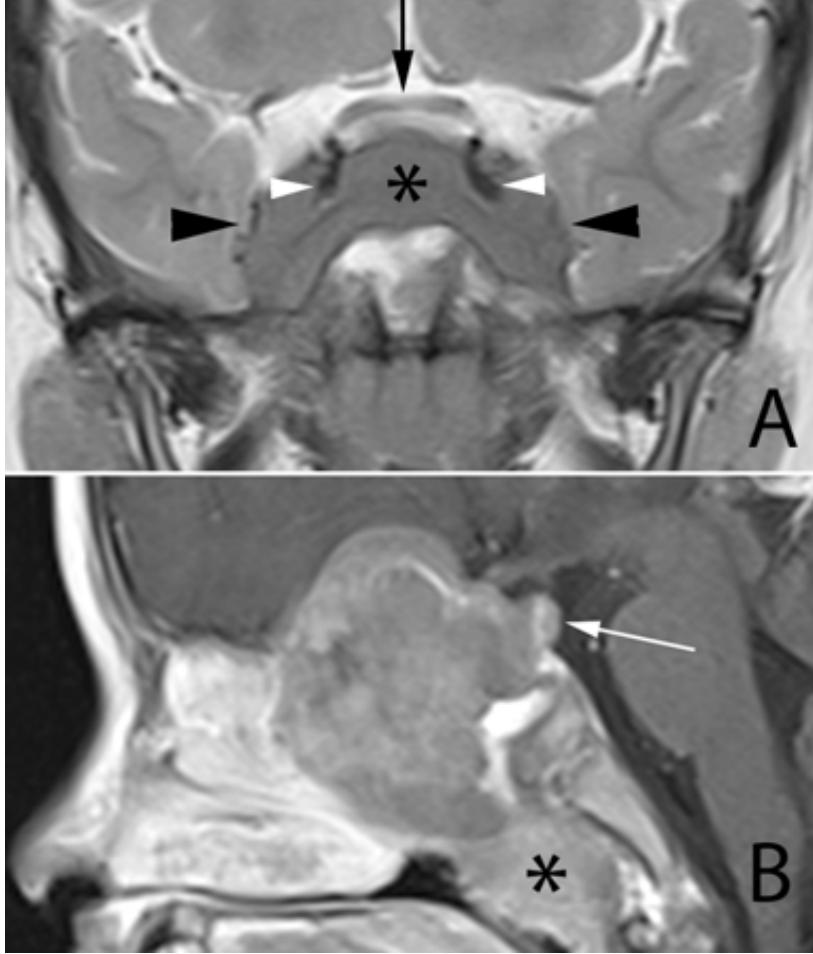

Fig. 3: (A) T2 weighted coronal MR image, slice positioned at the level of the optic chiasm (black arrow). Involvement of bilateral cavernous sinuses is evident, with tumour projecting into bilateral middle cranial fossae (black arrowheads). The cavernous internal carotid arteries appear encased (white arrowheads), and the pituitary fossa (asterisk) is occupied.

(B) Midsagittal T1 Gadolinium enhanced image shows the intracranial extent of the tumour, with its posterior margin inseparable from the pituitary fossa (white arrow). Involvement of much of the sphenoid sinuses and posterior ethmoid air cells is evident. Nasopharyngeal soft tissue (asterisk) of different signal intensity to the mass represents adenoid hypertrophy.

Subsequently, the patient underwent examination of nasal cavity under anesthesia and bifrontal craniotomy with tumor debulking. Intraoperative findings showed a large base of skull soft and gelatinous mass with epicenter at the ethmoid and it infiltrates the olfactory bulbs. Examination of nasal cavity revealed a midline smooth bulging mass arising superiorly from skull base and it occupies bilateral sphenoethmoidal recess but the mucosa appeared intact.

The tissue was sent for histopathological examination and the microscopic findings were consistent with Burkitt's Lymphoma.

Based on these findings, we established the final diagnosis of Burkitt's Lymphoma of base of skull and bilateral optic neuropathy with orbital apex syndrome. She was planned for MRI whole body for staging of tumor followed by chemotherapy. Unfortunately, due to financial constraint, family opted for continuation of management in her country. She was discharged 12 days post operatively. However patient's general condition rapidly deteriorated and succumbed 2 months after the initial presentation. 


\section{DISCUSSION}

The Endemic variant Burkitt's Lymphoma has a preponderance in malaria endemic area. Malarial infected B-cell will interact with EBV to promote tumerogenesis ${ }^{[1]}$. To our knowledge, Aceh, Indonesia is endemic for malaria, a place where the patient originates $^{[5]}$.

The presentation of Burkitt's Lymphoma is highly dependent on the primary site of the tumor ${ }^{[4,6]}$. In our patient, the primary tumor is located at anterior skull base, ethmoid, sphenoid and it has effaced the bilateral optic nerve, hence explaining the rhino and neuroophthalmologic presentation. Imaging is the only modality available for the provisional diagnosis in such presentation. With the MRI findings, the diagnosis olfactory neuroblastoma with differential of craniopharyngioma was on top of the list. Histopathological examination is mandatory for the final diagnosis of $\mathrm{BL}$ to be made.

In this patient, the aim was to attain tissue diagnosis and complete tumor removal. Radiological assessment showed extension of tumor to bilateral cavernous sinuses and encasing the internal carotid arteries (Fig 3). These findings appear to be the limiting factor for endonasal endoscopic approaches ${ }^{[9]}$. Hence, this explained the transcranial approach in debulking the tumor. Apart from that, transcranial approaches is preferable in base of skull tumors $>4 \mathrm{~cm}$ in diameter or $80 \mathrm{~cm} 3^{[9]}$.

The disease-containing tissue can only be obtained via open surgery where the humongous tumor bulk is located at the base of skull. The rarity of the primary extranodal presentation and the difficulty in obtaining the tissue for examination has made the diagnosis challenging, resulting in delayed in making the diagnosis. To date, on PubMed database there were only two reported cases for Primary Burkitt's lymphoma of the skull base which share the same presentation as our patient ${ }^{[7,8]}$. In one of the cases, the visual loss is restored after an immediate endoscopic transnasal optic nerve decompression and biopsy followed by chemotherapy ${ }^{[8]}$.

Burkitt's Lymphoma is highly sensitive to chemotherapy. The combination of cyclophosphamide, vincristine, methotrexate, and prednisolone is the current mainstay chemotherapy and it has yielded 90\% long-term survival in patients limited to head and neck disease. Chemotherapy is highly toxic and the risk of tumor lysis is high in the initial cycles resulting in treatment related mortality (TRM). Hence, a well equipped supportive care is needed for a better care ${ }^{[1]}$.

Since such facilities are not readily available in low-income countries, the execution of intensive treatment may result in treatment-related mortality.
Thus, the degree of treatment needs to be tailored to the availability of supportive care, child tolerance to chemotherapy and the extent of co-morbidities. In the low-income countries, failure of treatment can be due to late presentation, progression of disease, treatment related mortality or by incomplete treatment[ 1]. In all, these factors can be demonstrated in our patient whereby financial burden and lack of supportive care may be the main limiting factor contributing to her untimely demise.

\section{CONCLUSIONS}

In conclusion, Burkitt's Lymphoma should be considered as one of the differential diagnosis in pediatric patient with rapid neuro-ophthalmological deterioration especially in regions where malaria is endemic. The aggressiveness of the disease at base of skull warrants early tissue diagnosis by means of surgical intervention to ensure prompt commencement of treatment.

\section{CONFLICT OF INTEREST}

There are no conflicts of interest.

\section{REFERENCES}

1. Molyneux EM, Rochford R, Griffin B, et al. Burkitt's lymphoma. In: The Lancet. Vol 379. ;2012:1234-1244.

2. Burkitt D. Etiology of Burkitt's lymphoma - an alternative hypothesis to a vectored virus. J Natl Cancer Inst 1969; 42:19-28.

3. Brady G, MacArthur GJ, Farrell PJ. Epstein-Barr virus and Burkitt lymphoma. Postgrad Med J. 2008;84(993):372-377.

4. Derinkuyu BE, Boyunağa Ö, Öztunalı Ç, Tekkeşin F, Damar Ç, Alımlı AG, Okur A. Imaging features of Burkitt lymphoma in pediatric patients. Diagnostic and Interventional Radiology. 2016 ;22(1):95.

5. Ekawati LL, Herdiana H, Sumiwi ME, et al. A comprehensive assessment of the malaria microscopy system of Aceh, Indonesia, in preparation for malaria elimination. Malar J. 2015;14(1).

6. Nikgoo A, Mirafshariyeh SA, Kazeminezhad B, Eshkevari PS, Fatemitabar SA. Burkitt's Lymphoma of Maxillary Sinuses: Review of Literature and Report of Bilateral Case. J Oral Maxillofac Surg. 2009;67(8):1755-1763.

7. Kalina P, Black K, Woldenberg R. Burkitt's lymphoma of the skull base presenting as cavernous sinus syndrome in early childhood. Pediatr Radiol. 1996;26(6):416-417. 
8. Emami N, Daniel SJ. Acute bilateral blindness as a presenting symptom of Non-Hodgkin's lymphoma. Int J Pediatr Otorhinolaryngol. 2012;76(5):740-741.
9. Fraser JF, Nyquist GG, Moore N, Anand VK, Schwartz TH. Endoscopic endonasal transclival resection of chordomas: operative technique, clinical outcome, and review of the literature. Journal of neurosurgery. $2010 ; 112(5): 1061-9$. 